\title{
Antimicrobial nanolayered and nanofibrous metal phosphates for prospective biomedical applications
}

\author{
A. Adawy ${ }^{1^{*}}$, Z. Amghouz ${ }^{2}$, C. Trobajo ${ }^{3}$, J. R. García ${ }^{3}$ \\ ${ }^{1}$ Unit of Electron Microscopy and Nanotechnology, Institute for Scientific and Technological Resources (SCTs), University of Oviedo, \\ 33006 Oviedo, Spain, \\ ${ }^{2}$ Department of Material Science and Metallurgical Engineering, University of Oviedo, 33203 Gijón, Spain, \\ ${ }^{3}$ Department of Organic and Inorganic Chemistry, University of Oviedo, 33006 Oviedo, Spain
}

hassanalaa@uniovi.es

Synthesizing pharmaceuticals and biomaterials that have antimicrobial biofunctionality has gained an increasing interest. In this respective, silver nanoparticles (AgNPs) possess outstanding antimicrobial activity. Nevertheless, their uncontrolled release in biological media can induce cytotoxic effects. In order to improve their bio-functionality, a number of metal phosphates, based on titanium and zirconium as the metallic sources, belonging to two distinct morphologies: nanolayered and nanofibrous structures were used as reservoirs for AgNPs (Fig.1). Nanolayered $\alpha$-phases of titanium- and zirconium (IV) phosphates were supplemented with AgNPs. The structural assessment confirmed the stability of the structures and their sizes that laid in the nanoscale at least in one dimension. The cytocompatibility assays confirmed the biocompatibility of the pristine phases and the antimicrobial assays confirmed that both silver-enriched nanolayered structures maintain an antibacterial effect at reasonably low concentrations. The silver release in these layered structures is largely controlled owing to their intercalation [1]. On the other hand, the nanofibrous metal phosphates were utilized through synthesizing two phases of nanostructured titanium phosphate ( $\pi$ and $\rho$ polymorphs). To assess the feasibility of using these nanofibrous $\pi$ and $\rho$ titanium (IV) phosphate phases for antimicrobial applications, they were enriched with AgNPs. The antimicrobial assays confirmed their functionality as antimicrobial materials. Moreover, the silver release could be controlled through enriching these nanofibrous Ag-enriched structures with strontium that increased their cytocompatibility, as was confirmed using the cytocompatibility and ion-release assessments. As a direct application of these phases for biomaterials applications, Ag-Sr-enriched nanostructured $\pi$-titanium phosphate was induced to grow on a commercially available titanium alloy (Ti-6Al-4V), widely used in orthopedic and dental implants. The structural and microscopic observations confirmed the resultant phases and their enrichment with strontium and AgNPs. Analysis of the surface roughness revealed that its values lay at the interface between the nanosized and micro sized topologies [2]. The results altogether demonstrate the feasibility of using the studied (Sr-) Ag-enriched layered and fibrous metal phosphates as bio-functional bone cement/filling or coatings for metallic implants for biomedical applications.

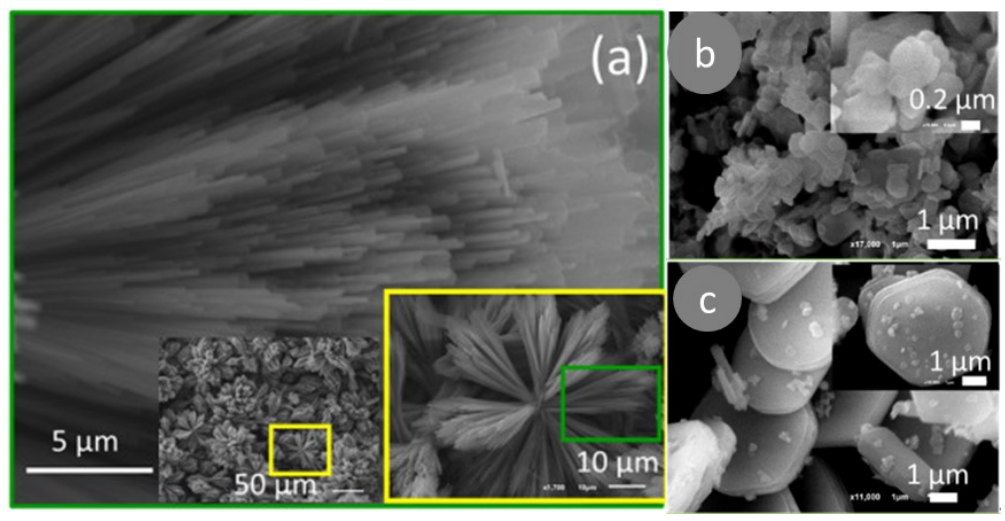

Figure 1. SEM images for (a) nanofibrous $\pi$-titanium phosphate, (b) nanolayered $\alpha$-titanium phosphate $\&$ (c) $\alpha$-zirconium phosphate.

[1] García, I., Trobajo, C., Amghouz, Z., Alonso-Guervos, M., Díaz, R., Mendoza, R., Mauvezín-Quevedo, M. \& Adawy, A. (2021). Mater. Sci. Eng. C, 126, 112168.

[2] García, I., Trobajo, C., Amghouz, Z. \& Adawy, A. (2021). Materials, 14, 1481.

Keywords: Biomaterials, Antimicrobial Silver, Titanium Phosphate, Strontium, Electron Microscopy

This research was funded by MINECO, grant number MAT2016-78155-C2-1-R and by the Government of the Principality of Asturias, grant number GRUPIN-IDI/2018/170. Special thanks go to S. Garcia-Granda for the continuous support. 